\title{
MUSCLE ACTION OF THE VERTEBRAL COLUMN
}

\section{P.H Newman, D.S.O., M.C., F.R.C.S.}

The spine and its associated musculature can be regarded from an engineering viewpoint, and multiple analogies between structures and functions of the human spine and those, for example, of the mast of a yacht or the jib of a crane can be made to illustrate this.

The spine has four main functions (Fig. 1). The first is that of a vertical supporting structure or mast from which movements of the upper limbs may be performed. Secondly, the spine exists as a structure which resists downwards thrusts, namely a strut. Its third function is to act as a cantilever, being cantilevered from the sacrum as when a coal-miner is stooping in a low seam. In addition its fourth function is that of a mobile structure represented by a segmented duct. This is not analogous to a mast as 24 segments have to be controlled. For this purpose the vertebral bones are designed with a main body and a series of controlling levers posteriorly.

Dealing with the first function of the spine, lateral stability is maintained, as in a yacht, by using a system of stays, for neither the spine nor the mast can be sunk very far into their respective supports (Fig. 2). In the spine the stays are represented by muscles attached between the shoulder girdle, spine, and pelvic girdle. In fact there is a geometrical similarity between the mast and the spine in that each is sunk to a depth of one eighth of its length into the base; a common practice in civil engineering, an example being that the Post Office Tower has a restraining collar at approximat ely one eigth of its total distance above the ground (Fig. 3).

Downward thrusts are induced in the spine by the weight of the structures above and by the downward pull of the controlling muscles, which is resisted by the spine in its capacity as a strut (Fig. 2). Although there is no direct comparison between spine and mast in this sense, muscular contraction can be equated with the tightening of stays of the mast inducing greater thrust in the spine and mast respectively. Therefore the magnitude of the total thrust in the spine is over and above that attributable solely to the weight of the structures of the upper body. This thrust is magnified during the adolescent growth spurt where the skeleton grows at a faster rate than its associated musculature and in these conditions postural abnormalities such as adolescent kyphosis or scoliosis are liable to increase. 

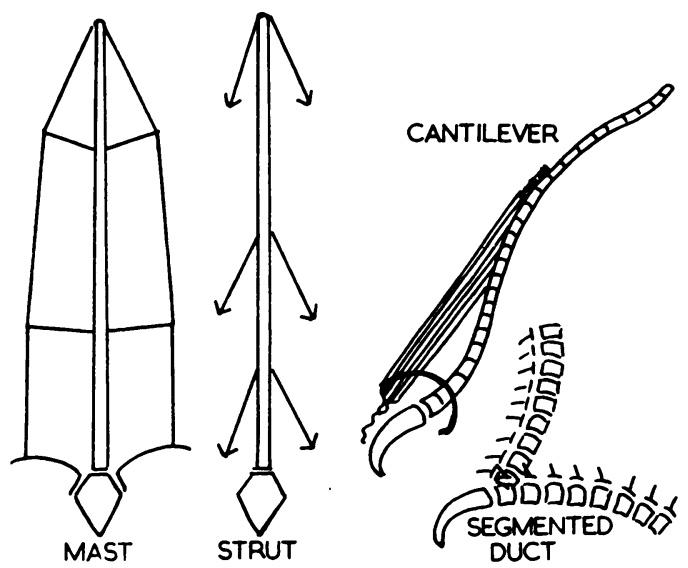

Fig. 1.

The four main functions of the spine.
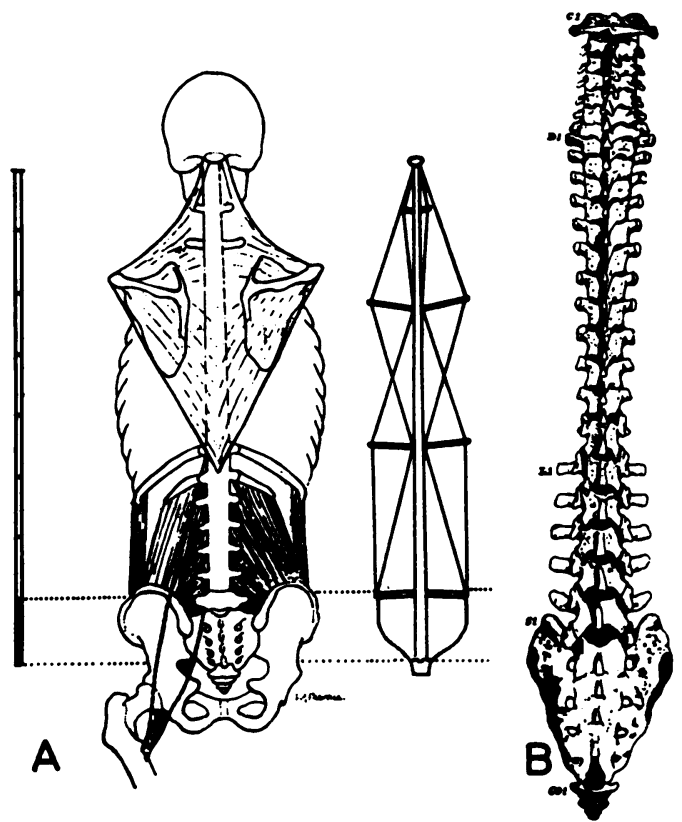

Fig. 2.

Geometrical relationship between the spine and mast indicating the depth to which the lower end is sunk into the base.

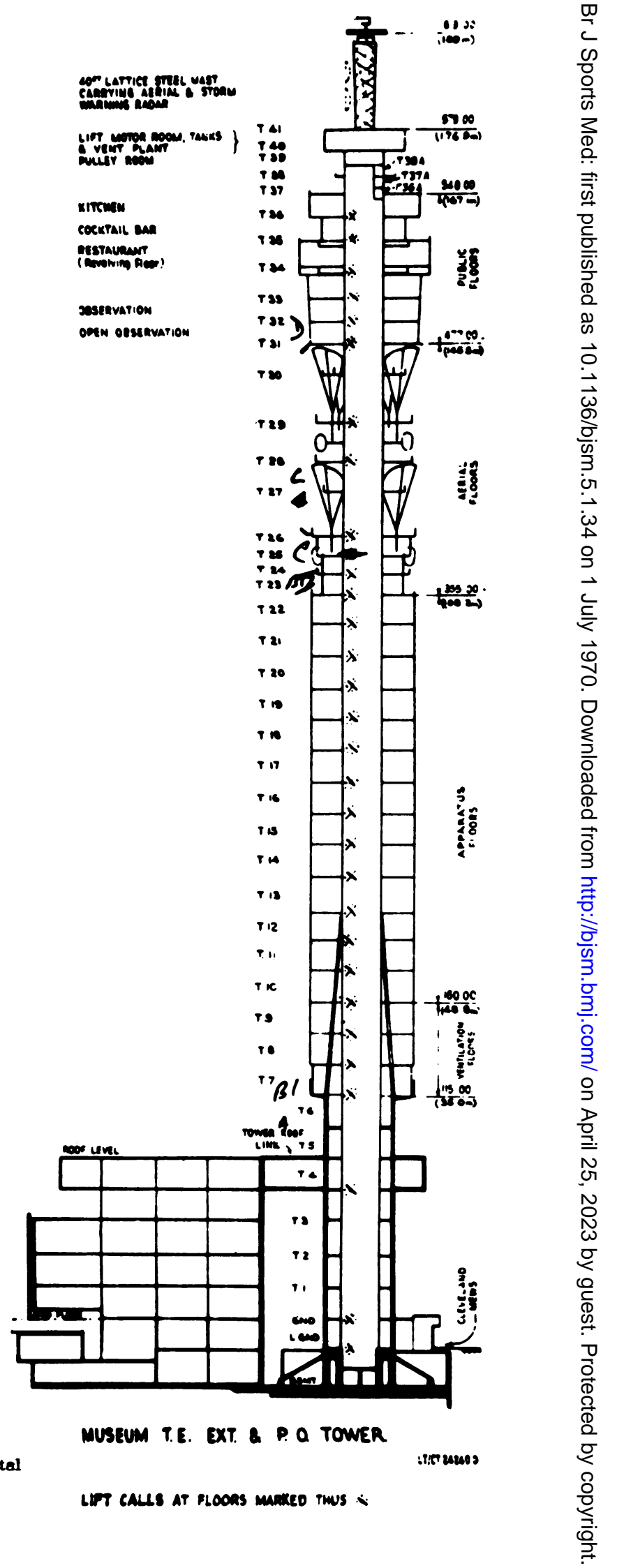


It is in the fore and aft staying that a great difference appears between the mechanism of the yacht's mast and the spine. The length of the yacht gives a large base so that the back stay can be attached far away from the foot of the mast. In the spine this is not apparently so, the sacrospinalis muscle and the lumbar fascia are attached close to the base, but not so close as would first appear because the posterior enlarged end of the iliac crest is set three or four inches posterior to the nucleus pulposus of the lumbosacral disc. This anatomical arrangement is equivalent to the cathead of a crane whi ch separates the stabilising cable from the foot of the derrick.

The spine has to work in various postures so that in positions other than the vertical it acts as a cantilever with the point of maximum compression on the lumbar vertebral bodies and discs and the point of maximum tension at the attachment of the muscles, fascia and ligaments posteriorly.

In weight lifting, the spine must be kept rigid by contraction of the psoas, quadratus lumborum and sacrospinalis muscles, the initial stages of the lift being performed by gluteus maximus and the extensors of the knee. The mechanisms of the forces induced at the base of the spine are referred to in Fig. 4.

In the later stages of the lift the relationships between individual segments of the spine are changed (Fig. 5), and the point at which this occurs is that at which patients with disorders of the spine wriggle in order to produce spinal extension. Changes in the relationship between individual segments are produced by intrinsic muscles of the spine such as multifidus and interspinalis.

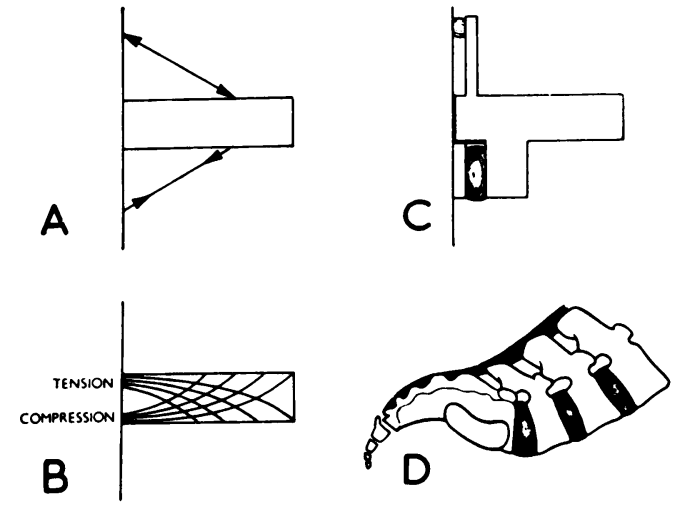

Fig. 4 .

Forces induced in the spine in the 'cantilever' situation.
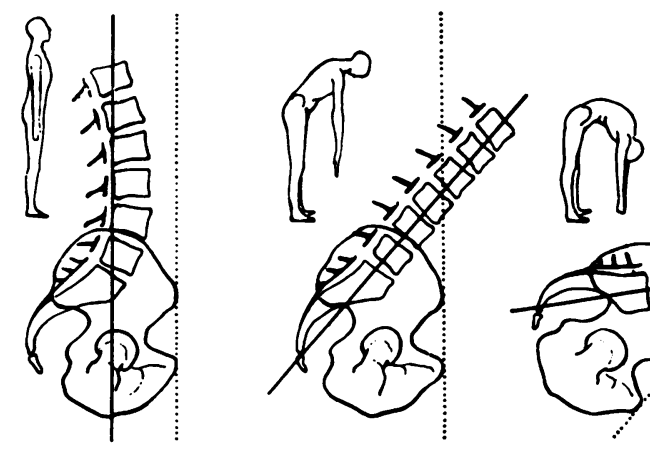

Fig. 5 .

Segmental relationships in the lumbar spine at three stages of spinal extension.

The figures are reproduced by courtesy of the Editor of the Proceedings of the Royal Society of Medicine from: Newman P.H. 1968, Proc. Roy. Soc . Med. 61 35-41. 